\title{
Comparative Study between Protective Effects of L-Carnitine and Curcumin on \\ Nephrotoxicity Induced by Cisplatin in Adult Male Albino Rats
}

\section{Noha Aboelnaga Ashour a, Dorreia Abd Allah Mohamed ${ }^{b}$, Maha Abd Elbaki Ahmeda}

a Human Anatomy and Embryology Department, Faculty of Medicine, South Valley University ,Qena, Egypt.

b Human Anatomy and Embryology Department, Faculty of Medicine, Assiut University, Assiut, Egypt.

\begin{abstract}
Background: Cisplatin is regarded as a main anticancer drug. However, nephrotoxicity is its major side effect and chief dose-limiting factor. L-carnitine (LC) is a natural nutrient, while Curcumin (CMN) is derived from turmeric (Curcuma longa) plants. Both could prevent nephrotoxicity.
\end{abstract}

Objectives: To evaluate nephrotoxicity induced by cisplatin , detect protective effect of L-carnitine and curcumin in reducing cisplastin nephrotoxicity and determine which is more potent .

Materials and Methods: Forty adult male albino rats were randomly divided into 4 groups ten rats each; Group1; Control group, received isotonic saline, 2. Group 2; cisplatin treated $(7 \mathrm{mg} / \mathrm{kg}$ body weight as a single dose), Group 3; treated with LC (500 $\mathrm{mg} / \mathrm{kg} /$ day) plus cisplatin, Group 4; treated with cisplatin plus CMN $(100 \mathrm{mg} / \mathrm{kg}$ /day).The duration of experiment was 10 days. Specimens were prepared for light and electron microscopic examination. Morphometric and statistical studies were done to measure the diameter of proximal convoluted tubules (PCT) and Bowman's space.

Results: The cisplatin-treated rats had destructive damage of the renal glomeruli including all types of pathological changes in the cortex and marked degeneration of the proximal convoluted tubules (PCTs). The treatment with LC and curcumin prevented severe damage caused by cisplatin. However LC treated rats showed more improvement than CMN treated group.

Conclusions: Administration of L-carnitine with cisplatin decreases its nephrotoxicity which is the major cisplatin side effect.

Keywords: Kidney, Cisplatin, L-carnitine, Curcumin.

Copyright: (c) Ashour et al (2022) Immediate open access to its content on the principle that making research freely available to the public supports a greater global exchange of knowledge. Users have the right to Read, download, copy, distribute, print or share link to the full texts under a Creative Commons BY-NC-SA 4.0 International License. 


\section{Introduction}

Cisplatin (CDDP) is a famous effective DNA intercalating anticancer drug. It is used with other chemotherapy in treatment of a variety of tumors such as gonadal cancers, tumors of the head and neck, tumors of the uterus, mesothelioma, lung cancer, urothelial carcinoma and cancers of the gastrointestinal tract (Jafri et al. , 2010).

Ototoxicity, nephrotoxicity,neurotoxicity and bone marrow suppression are common side effects of cisplatin, but its major dose- limiting side effect is nephrotoxicity (Istvan and Robert, 2003). The nephrotoxicity of CDDP is thought to be mediated by several mechanisms including DNA deterioration, oxidative stress, caspase energizing (extrinsic pathway of apoptosis), mitochondrial defects (intrinsic pathway of apoptosis), and finally cell death either by necrosis or Apoptosis, according to dose of it (Burne et al.,2001).

L-carnitine is a natural nutrient necessary for $\beta$-oxidation of fatty acid in mitochondria to generate ATP .It is synthesized internally from the essential amino acids lysine and methionine. It effectively prevents mitochondrial damage caused by oxidative stress through prevention of depletion of antioxidant system of the kidney (Abdulhakeem, 2007). Curcumin(CMN) is derived from turmeric (Curcuma longa) plants. It is a powerful antioxidant. It inhibits peroxidation by cleaning reactive free radicals involved in the peroxidation process(Trujillo et al.,2013).

In this study, we evaluated nephrotoxicity induced by cisplatin, protective effect of Lcarnitine and curcumin in reducing cisplastin nephrotoxicity and determine which is more potent?

\section{Materials and Methods \\ Chemicals}

CDDP was purchased from Sandoz Pharma company .LC was purchased from MEPACO company. CMN was obtained from Al-Haditha Pharma Company. All used reagents were of analytical grade and highest purity.

\section{Animals and Experimental design}

In this study, 40male albino Wister apparently healthy rats, 3-6 months of age, weighted $200 \pm 50 \mathrm{~g}$ were used. They were kept under a well-regulated schedule of light and dark ( $12 \mathrm{~h}: 12 \mathrm{~h})$ at $22-25^{\circ} \mathrm{C}$. The animal had free access to tap water and treated according to the guidelines of the Animal House of South Valley University-Qena, where standard commercial pellets were used for feeding and water ad libitum. Other conditions pertaining to the health of the animals were maintained during the duration of the experiment. All experimental protocols were performed in accordance with the local institutional guidelines and approved by the Animal Ethical Committee, South Valley University, Qena, Egypt. They were kept for at least 10-15 days prior to experimentation for adaptation

Duration of experiment was 10 days. The included rats were randomly divided into 4 equal groups (n $=10$ each).

- Group I (control)received isotonic saline

- Group II( CDDP group) : received cisplatin single dose $(7 \mathrm{mg} / \mathrm{kg})$, by intraperitoneal injection (Ugur et al.,2015).

- Group III( CDDP-LC) : received LC (500 $\mathrm{mg} / \mathrm{kg} /$ day) for 10 successive days .On the third day of experiment ,CDDP was administrated in the same way as group II(Cayir et al.,2009).

- Group IV (CMN + CDDP) : received $\mathrm{CMN}(100 \mathrm{mg} / \mathrm{kg} /$ day $)$ by gavage for 10 successive days .On the third day of the experiment ,CDDP was administrated in the same way as group II(Ugur et al.,2015).

\section{Samples' collection}

The animals were starved overnight at the end of the experimental phase and anaesthetized using diethyl ether inhalation; then, then the abdomen was opened and the kidneys were immediately dissected and adherent tissues were cleaned and immersed in neutral buffered formalin $10 \%$ for histopathological examination. 
Ashour et al (2022)

\section{Histopathologic examination}

Using traditional paraffin-embedding methods processing of the fixed renal tissues was carried out. Using a microtome, five $\mu \mathrm{m}$ thick parts were obtained from prepared paraffin blocks. Next, hematoxylin and eosin stained (Gamble,2008), and examined with light microscope. Additionally, histological technique for semi-thin and ultrathin renal tissue sections were performed (Ayache $\boldsymbol{e t}$ al., 2010), where semi thin sectioning at one $\mu \mathrm{m}$ renal specimens were trimmed with a razor blade and stained with $2 \%$ aqueous toluidine blue then dried on a hot plate at $40{ }^{\circ} \mathrm{C}$ and were examined by light microscope. While, ultrathin sectioning at 50 $\mathrm{nm}$ was done on cooper grids. Sections were examined by a transmission JEOLJEM-100CX II electron microscope and were photographed.

\section{Morphometric and statistical analysis}

The diameter of PCT and Bowman's space in all groups were measured (at magnification of $x 400$ Microscope).The previous parameters were measured using the software (ImageJ).Variables were represented by mean \pm standard deviation $(\mathrm{M} \pm \mathrm{SD})$. Data analysis using t-test and one-way ANOVA test of SPSS 22 was performed to calculate $\mathrm{p}$ value which was considered significant if $p<0.05$.

Results

Light microscopic result $\mathrm{H} \& \mathrm{E}$ and toluidine blue semithin sections: figures 1,2

A) Control Group (Group I) (figure 1, 2 A\&B): They showed the normal renal cortex structure which is formed of renal corpuscles, PCTs and DCTs. They showed the normal appearance of the glomerulus, mesangial cells, Bowman's space and vascular pole.

B) CDDP Group (Group II) (Figure 1, 2 C \& D): Destructive damage of renal cortex was noticed. Shrinkage and atrophy of renal glomeruli was present with congestion of glomerular capillaries, widening of Bowman's space and thickening of glomerular basement
SVU-IJMS, 5(1):331-342

membrane . PCTs showed tubular degeneration, intraluminal casts, epithelial desquamation, nuclear pyknosis and vacuolar formation .

C) CDDP-LC Group (Group III) (Figure 1, 2 E\&F): They showed relative regain of the normal renal structure and reversion of most of the destructive changes. Most tubules regain their normal lining. There was decreased glomerular damage with more or less normal urinary space.

D) CDDP-CMN Group (Group IV) (Figure1, 2 G\&H): They showed partial restoration of the normal renal architecture, however, mild glomerular and tubular damage were still noted.

\section{Electron Microscopy Results (Figure3)}

A) Control Group (Group I) (Figure 3 A\&B): They showed structure of renal corpuscles that are characterized by a Bowman's capsule enclosing a tuft of capillaries. Normal renal filtration barrier was present that consists of glomerular basement membrane, endothelium which is fenestrated and secondary foot processes of podocyte separated by filtration pores .PCTs had apical numerous microvilli, euochromatic rounded nucleus, lysosomes, RER and basal membrane invaginations associated with elongated mitochondria.

B) CDDP Treated Group (Group II) (Figure 3 C\&D): They revealed destructive damage of renal cortex. The renal glomeruli showed congested glomerular capillaries with irregular basement membranes. The podocytes appeared damaged with having large intraluminal vacuoles, partial loss of basal infoldings and condensed nucleus.Some PCTs appeared pleomorphic with swollen 
mitochondria, many lysosomes, disintegrated microvilli, necrotic epithelial cells and a large number of translucent vacuoles.

C) CDDP-LC Group (Group III) (Figure 3 E\&F): There was relative repair of the renal structure. of the renal structure. They showed reestablishment of the solidity of the apical brush border, more or less normal mitochondria arranged basally with restored basal infoldings, and relatively normal nuclei. However, the glomeruli showed mild degenerative changes as mild BM thickening, mild capillary congestion and some podocytes degenerative changes.

D) CDDP-CMN Group (Group IV) (Figure 3 G\&H): They showed relative regeneration of the normal renal architecture. The tubules began regaining its lining with apical brush border. Some swollen mitochondria disappeared and others still present. More or less normal nuclei are seen. Still mild degenerative changes as mild thick irregular BM , capillary congestion and mild degenerated podocytes are noticed.

\section{Morphometric results (Figure 4, 5)}

Acquired changes showed powerful relationship between histological and morphometric variation in Cisplatin nephrotoxicity.

Morphometric analysis of tubules revealed that the diameters of PCT were different in cisplatin group proving statistically high significant increase in their diameter. PCT is the first site affected by cisplatin due its aggregation in this part of nephron. Morphometric analysis of the glomeruli indicated that in the the cisplatin group they showed high significant increase in the size of Bowman space.

Table 1. Diameters of PCTs and Bowman's space

\begin{tabular}{|l|l|l|l|l|}
\hline Diameters & $\begin{array}{l}\text { Group } \\
\text { I }\end{array}$ & $\begin{array}{l}\text { Group } \\
\text { II }\end{array}$ & $\begin{array}{l}\text { Group } \\
\text { III }\end{array}$ & $\begin{array}{l}\text { Group } \\
\text { IV }\end{array}$ \\
\hline PCT & & & & \\
$(\mathrm{M} \pm$ SD) & $\begin{array}{l}7.6 \pm \\
0.6\end{array}$ & $\begin{array}{l}20.8 \pm \\
1.8\end{array}$ & $\begin{array}{l}9.7 \pm \\
0.66\end{array}$ & $\begin{array}{l}11.4 \pm \\
1.09\end{array}$ \\
\hline $\begin{array}{l}\text { Bowman's } \\
\text { space (M } \pm\end{array}$ & $11.52 \pm$ & $18.63 \pm$ & $12.8 \pm$ & $14.32 \pm$ \\
SD) & 0.9 & 1.02 & 1.3 & 0.89 \\
\hline
\end{tabular}

Table 2. Diameters of PCT Bowman's space comparing control and CDDP treated group

\begin{tabular}{|l|l|l|l|}
\hline Diameter & Control & CDDP & P-value \\
\hline $\begin{array}{l}\text { PCT }(\mathrm{M} \pm \\
\text { SD) }\end{array}$ & $7.61 \pm .65$ & $20.82 \pm 1.82$ & $\mathbf{0 . 0 0 1 *}$ \\
\hline $\begin{array}{l}\text { Bowman's } \\
\text { space (M } \pm \\
\text { SD) }\end{array}$ & $11.52 \pm .92$ & $18.64 \pm 1.03$ & $\mathbf{0 . 0 0 1 *}$ \\
\hline
\end{tabular}

Table 3. Diameters of PCT Bowman's space comparing CDDP- LC and CDDP-CMN treated group

\begin{tabular}{|l|l|l|l|}
\hline Diameter & $\begin{array}{l}\text { CDDP + } \\
\text { LC }\end{array}$ & $\begin{array}{l}\text { CDDP + } \\
\text { CMN }\end{array}$ & P-value \\
\hline PCT (M \pm SD) & $9.74 \pm .66$ & $11.43 \pm 1.09$ & $\mathbf{0 . 0 0 1 *}$ \\
\hline $\begin{array}{l}\text { Bowman's } \\
\text { space (M } \pm \text { SD) }\end{array}$ & $12.8 \pm 1.32$ & $14.33 \pm .9$ & $\mathbf{0 . 0 0 8 *}$ \\
\hline
\end{tabular}




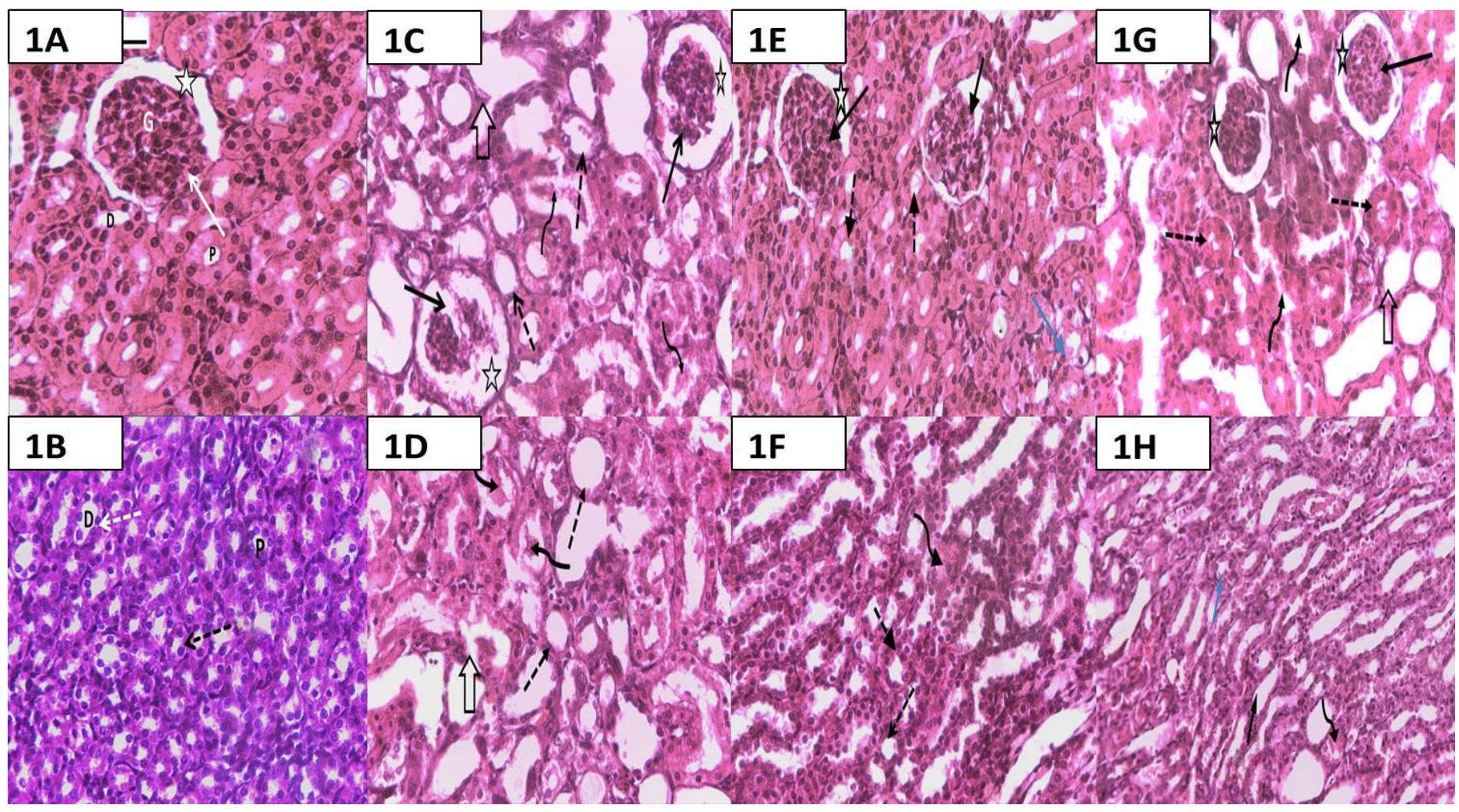

Fig. 1.Photomicrograph of the histopathological findings of the kidneys of the studied groups of H\&E stained tissue sections using light microscopy $(\mathbf{X 4 0 0 ) . 1 A}$. Cortex of kidney from the control group (group I) showing normal kidney parenchyma; glomerulus (G), PCT (P), DCT (D), Bowman's space (star) and vascular pole (white arrow). 1B. Renal medulla from (group I) revealing PCT (P) which have narrow lumen and lined by simple columnar cells (black dotted arrow) and DCT (D) which have faint acidophilic cytoplasm and lined by cuboidal cells (white dotted arrow). 1C. Renal cortex from the CDDP group (group II) showing shrunken lobulated glomeruli (black arrow), obvious wide Bowman's space (star), severe tubular dilation and degeneration (black dotted arrow) with areas of degeneration (open arrow) and intraluminal casts (curved arrow). 1D. Renal medulla from (group II) showing severe tubular dilation and degeneration (black dotted arrow) with areas of degeneration (open arrow) and intraluminal casts (curved arrow). 1E. Renal cortex from CDDP-LC treated rat (group III) showing slight amelioration of the atrophied glomeruli (black arrow). More or less normal Bowman's space is noticed (star). The tubules are almost normal (black spotted arrow). Vacuolations in epithelium of some tubules are seen (blue arrow). 1F. Renal medulla from (group III) showing more or less normal tubules (black dotted arrow). Intraluminal cast is noticed (curved arrow). 1G. Renal cortex from CDDPCMN (group IV) showingnormal glomerular structure (black arrow), persistent dilated Bowman's space (star), normal tubules in some areas (black dotted arrow) and some dilated tubules with degenerated parts (open arrow). Also, there are still intraluminal casts (curved arrow). 1H. Renal medulla from (group IV) showing some dilated tubules (black arrow), others normal tubules (blue arrow) and still noted intraluminal casts (curved arrow). 


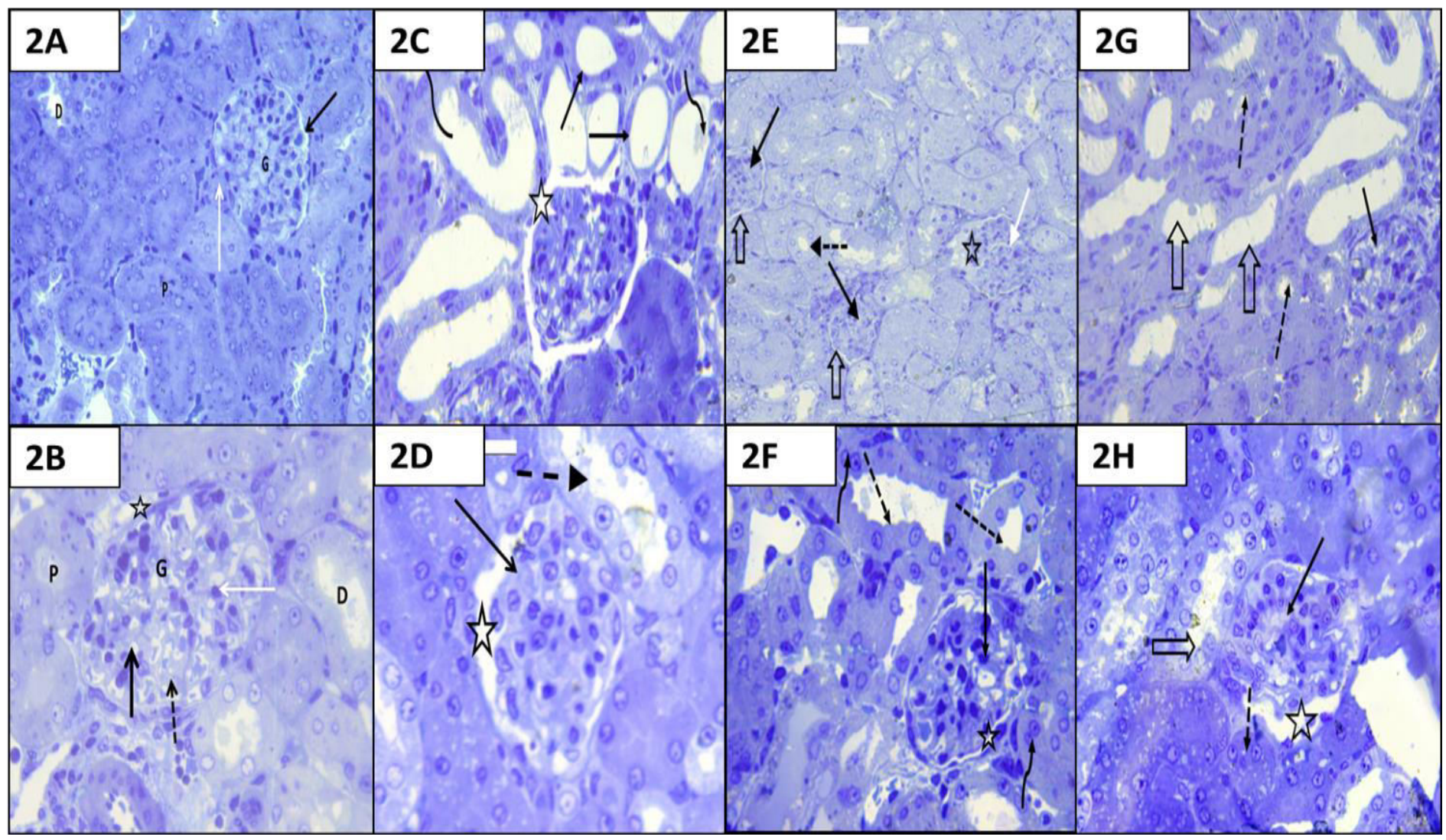

Fig .2. Photomicrograph of the histopathological findings of the kidneys of the studied groups using Semithin sections stained with toluidine blue $4000 \mathrm{X} \& 6300 \mathrm{X}$. 2A. Kidneys from the control group (group I) showing normal appearance of glomerulus (G), Bowman's space (black arrow), vascular pole (white arrow) PCT and DCT. 2B. Normal appearance of PCT (P), DCT (D), Bowman's space (star) and glomerulus (G) which has mesangial cells (white arrow), podocytes (black dotted arrow) and capillaries (black arrow).2C. represent kidneys from CDDP group (group II) showing tubular degeneration and dilatation (black arrows), intraluminal casts (curved arrow) and Bowman's space widening (star). 2D. A shrunken lobulated glomerulus (black arrow) with dilated Bowman's space (star). Vacuolated cells in the epithelial lining of tubules (black dotted arrow). 2E. represents kidneys from CDDP-LC group (group III) revealing normal tubules (black dotted arrow), some normal glomerular structure (black arrow), a lobulated glomerulus (red arrow), a normal Bowman's space (open arrow) and another dilated Bowman's space (star). 2F. Normal tubules (black dotted arrow), binucleated cells (curved arrow), normal renal glomerulus (black arrow) and almost normal Bowman's space (star). 2G. Kidneys from the CDDP+ CMN group (group IV ) revealing normal renal glomerulus (black arrow), some dilated tubules (open arrow) and others normal (black dotted arrow). 2H. Normal renal glomerular structure (black arrow), dilated Bowman's space (star), some irregularity in the lining of the tubules (open arrow) and others were normal (black dotted arrow). 

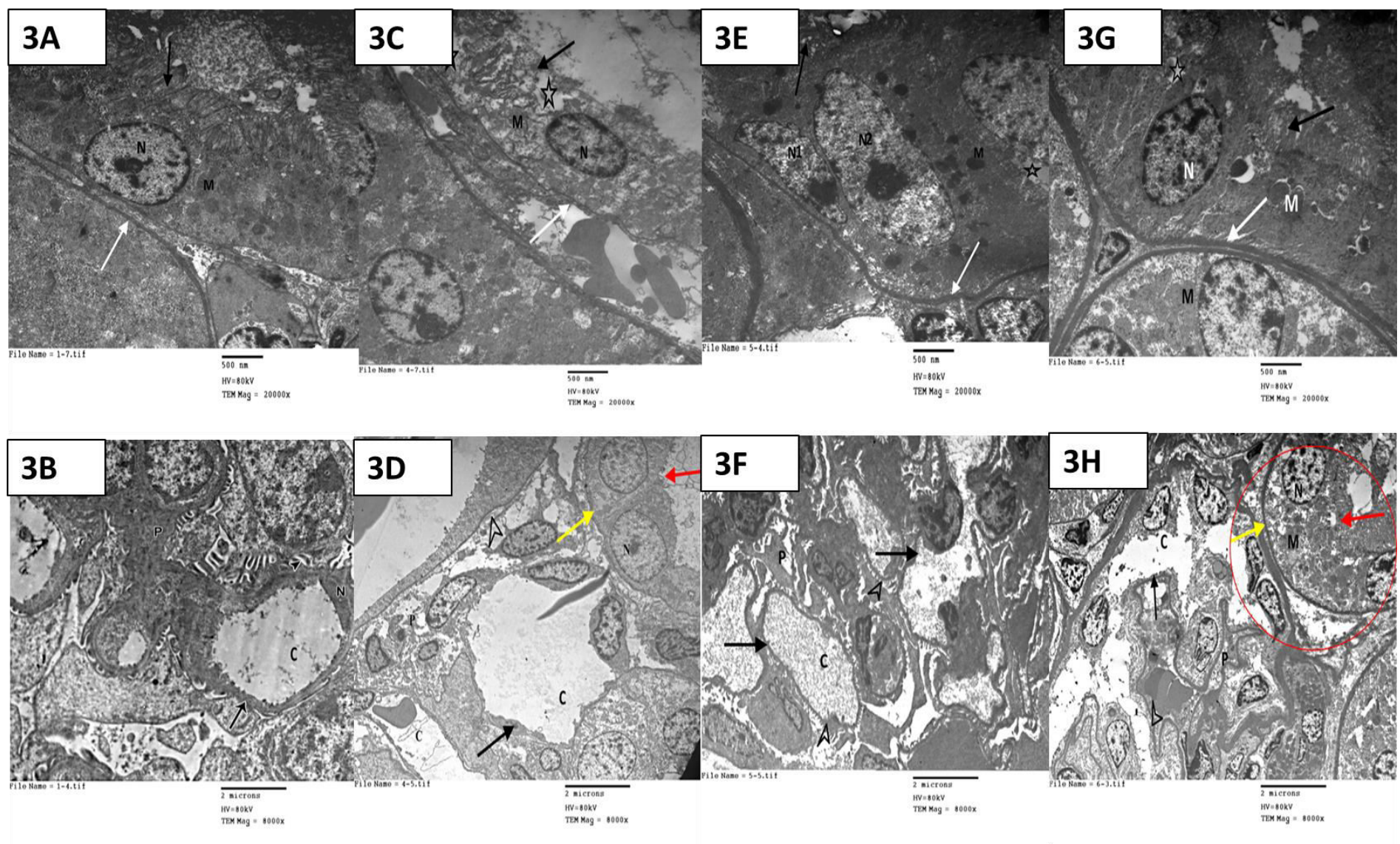

Fig .3. Photomicrographs of histopathological findings of the kidneys of the studied groups using electron microscopy. 3A. Kidneys from the control group (group I) in PCT revealing regular basal membrane (white arrow), euochromatic large rounded nucleus $(\mathrm{N})$, many elongated basally arranged mitochondria $(\mathrm{M})$ and regular apical microvilli (black arrow). 3B. Revealing glomerular capillary within the glomerulus $(\mathrm{C})$, podocytes $(\mathrm{P})$ with its processes (arrow head) and normal glomerular basement membrane (black arrow ). 3C. Kidneys from the CDDP group (group II) revealed irregular basal membrane in PCT(white arrow), shrunken nucleus (N), swollen mitochondria (M), vacuolation (star) and disintegrated apical microvilli (black arrow).3D.Reveals wide glomerular capillary (C), damaged podocytes (P) with atrophy of its processes (arrow head) and irregular thick basement membrane of the glomerulus (black arrow). Irregular basal membrane of PCT (yellow arrow), irregular brush border (red arrow) and shrunken nucleus (N) are shown. 3E represents kidneys from CDDP- LC group (group III), PCT showing regular basal membrane (white arrow), large oval nucleus (N2), normal mitochondria (M)and normal apical microvilli (black arrow).However, there are still vacillations (star) and condensed nucleus (N1). 3F. reveals normal capillary (C), normal basement membrane (black arrow) and normal podocytes (P) with its foot processes (arrow head). However, a focal thickening is seen (curved arrow). 3G. represents kidneys from the CDDP-CMN group (group IV)) with normal ovoid nuclei (N), normal basal membrane (white arrow), normal microvilli (black arrow), swollen mitochondria (M) and vacuolation still are present (star) 3H.normal glomerular capillary (C), irregular basement membrane (black arrow) and distorted podocytes (P) with partial loss of its podocytes (arrow head). Also normal PCT is noticed (red circle) with normal microvilli (red arrow). 
Ashour et al (2022)

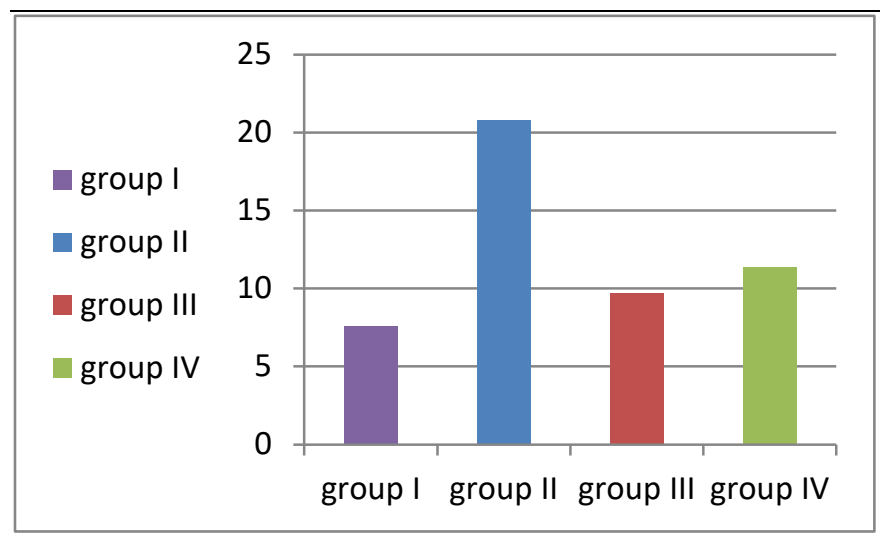

Fig .4. PCT diameters (mean) in all studied groups showed a significant widest diameters in CDDP (Group II). The measurements were significant due to $\mathrm{p}<0.05$.

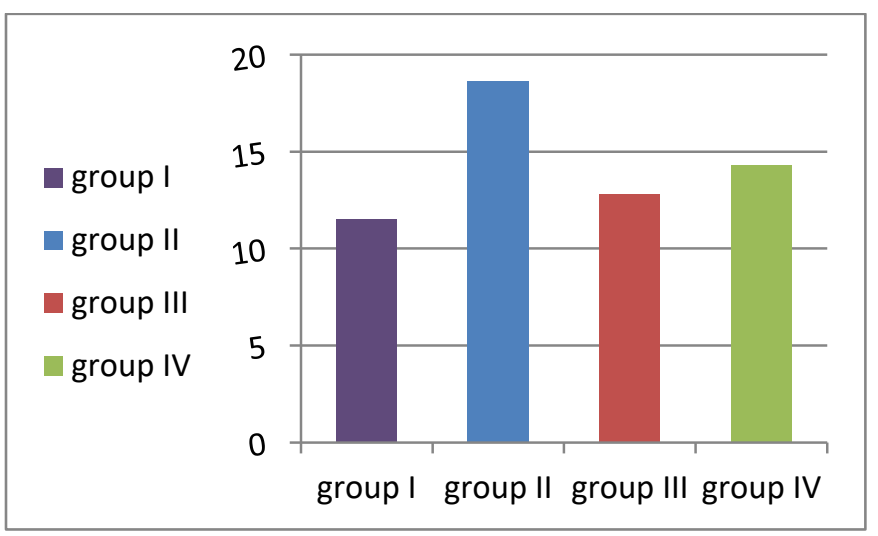

Fig .5. Bowman's space (mean) in all studied groups showed a significant increase in CDDP (group II). The measurements were significant due to $\mathrm{p}<0.05$.

\section{Discussion}

Cisplatin; CDDP (cis-diammine dichloroplatinum II) is one of the most efficient chemotherapeutic agents. Several tumors as head and neck, bladder, testicular, ovarian, uterine, cervical, non-small, and small- cell lung cancers are treated with cisplatin (Volarevic et al., 2019).

In the present study, the toxic impact of CDDP on adult albino male rats kidney and both protective and therapeutic role of LC and CMN on its nephrotoxicity were determined.

In the current study, injection of a single dose of cisplatin of $7 \mathrm{mg} / \mathrm{kg}$ (in group II ) (Ugur et
SVU-IJMS, 5(1):331-342

al.,2015) induced acute renal injury in rats as evidenced by histopathological examination and morphometric and analysis. The present work showed massive destructive damage of cortex of kidney including the glomeruli and the tubules. There were atrophy and shrinkage, congestion of glomerular capillaries, widening of Bowman's space, thick BM of glomeruli basement membrane with affection of the podocytes and dilated capillaries.

In accordance with the present work,(Ravindra et al.,2010; Mohamed et al.,2019; Rizk et al.,2019 ; Abd El-Kader and Taha, 2020 and Soheil et al., 2021) reported atrophy of some glomeruli with Bowman's spaces widening.

On contrary to the present work, (Yao et al.,2007 and Alhoshani, et al.,2017) reported that no effects occurred neither in the glomeruli structure nor the Bowman's space in rats treated with cisplastin..

These effects could be explained through the free radicals and ROS which are released causing oxidative damage and demolition of the structure of cells (Ajith et al., 2007;McSweeney et al.,2021).

The present work showed tubular degeneration and dilation, flattened epithelium, intraluminal casts, sloughed cells in lumen, vacuolation, condensed nuclei, some pleomorphic swollen mitochondria, many lysosomes and loss of brush border. These results are in inthe same context withthe previous studies (Ravindra et al.,2010; Rizk et al,. 2019;Abd El-Kader and Taha,2020;Abdel-Razek et al.,2020; Sadeghi et al., 2020 and Soheil et al., 2021).

Ravindra et al. (2010) and Johansson, (2014) claimed that the these degenerative changes that occurred in PCT is due to direct exposure of ROS and free radicals which leads to reduce the 


\section{Ashour et al (2022)}

area for active glucose reabsorption and consequences of renal failure.

LC is a low weight molecule. It is natural compound which gained from the diet and also is formed in vivo. The essential amino acids lysine and methionine are the components of it. It is necessary for long chain fatty acids into mitochondrial matrix which is very important for making energy(Yürekli et al., 2011).

CMN is a natural polyphenolic compound which contains diketone effective groups. CMN has many important effects as antioxidative, anti-inflammatory, anticancer and immunoregulatory effects (Gupta et al., 2012).

Many previous experimental studies reported that LC has protective action against the harmful effects of irradiation and anti tumor drugs including doxorubicin and cisplatin(Chang et al., 2002; SayedAhmed et al., 2004;Boonsanitet al., 2006; Tufekci et al., 2009).

In the current study, the effect of LC on nephrotoxicity induced by cisplatin was present .It has been proved that renal toxicity was improved with LC administration (Chang et al., 2002). But, a recent study deduced that there is no protective effect for LCandamifostin on kidney toxicity induced by cisplatin(Uzunoglu et al., 2010).In this work LC in doses of $500 \mathrm{mg} / \mathrm{kg}$ was used the to detect its protective effect on the kidney (group III).On LC administration ,there were improvement of pathological effects of cisplatin and the normal histological structure was regained. So the cisplatin nephrotoxicity could be get better by LC(Yürekli et al., 2011).

In the study determined that CMN reduced the toxic effects of cisplatin treatment, however there were still slight pathological findings which indicated by tubular degeneration and minimal congestion of interstitium. This suggests that CMN could improve the toxic action of cisplatin on kidney to some degree.

\section{SVU-IJMS, 5(1):331-342}

The phenolic structure and $\beta$-diketone content of CMN explain edits antioxidant properties (TopcuTarladacalisir et al., 2016; Ahmed et al., 2017; Kumar et al.,2017). (Waseem et al.,2013) found that alleviation of ROS in nephrotoxicity caused by cisplatin was due to CMN.(Kumar et al.,2017)found that pre-treatment of CMN before the use of Cisplatin increased a renoprotective action. They also found that post-treatment of CMN after the use of Cisplatin had no a renoprotective action. Topcu-Tarladacalisir et al.( 2016) explained the protective action of CMN due to prevention of oxidative stress, inflammation and apoptosis. We used dose of $100 \mathrm{mg} / \mathrm{kg}$ to assess its renoprotective effect (group IV) was used in the current study.

In this study, we further estimated the diameters of PCT and capsular space (Bowman's space). Both PCT and capsular space diameters were significantly increased in the cisplatin treated group in comparison to the control group. The LC treated group (group III) and CMN treated group (group IV) showed high significant decline. However, the LC treated group showed values more close to the normal values reported in the control group. These clues are in harmony with the results of Ahmed and Fouad (2019) study, and confirmed the protective role of LC and CMN, with superiority of the LC protective effect. This result is keeping with results found by Nameni and Nuranipilehrud (2019) who reported higher antioxidant activity of L-carnitine when compared with CMN. However, the variation did not score a significant degree. The results of (Abdel-Ghaffar et al.,2013) study are disconcordant with ours, as they concluded significantly higher antioxidant effect of CMN in comparison with LC. This may be explained by differed doses, as they used a dose of $200 \mathrm{mg} / \mathrm{kg}$, while ours was $100 \mathrm{mg} / \mathrm{kg}$.

\section{Conclusion}


Ashour et al (2022)

In the present work, administration of Lcarnitine with cisplatin decrease its nephrotoxicity which is the main side effect of cisplatin .

\section{References}

- Abd El-Kader M, Taha R(2020). Comparative nephroprotective effects of curcumin and etoricoxib against cisplatininduced acute kidney injury in rats. ActaHistochem, 122 (4): 151534.

- Abdel-RazekE A N, Abo-Youssef A M ,Azouz AA (2020).Benzbromarone mitigates cisplatin nephrotoxicity involving enhanced peroxisome proliferator-activated receptor-alpha (PPAR- $\alpha$ )expression. Life Sciences, 243:117272.

- Ahmed AY, Gad A M , El-Raouf O MA(2017).Curcumin ameliorates diclofenac sodium-induced nephrotoxicity in male albino rats. Journal of biochemical and molecular toxicology, 31(10): e21951.

- AjithTA, UshaS ,NivithaV (2007). Ascorbic acid and $\alpha$-tocopherol protect anticancer drug cisplatin induced nephrotoxicity in mice: a comparative study. Clinicachimicaacta, 375(1-2):82-86.

- Alhoshani AR, HafezMM, HusainS, AlSheikhAM, AlotaibiMR, Al Rejaie S S, et al.(2017). Protective effect of rutin supplementation against cisplatin-induced Nephrotoxicity in rats. BMC nephrology, 18(1): 1-10.

- Al-MajedA A(2007).Carnitine deficiency provokes cisplatin-induced hepatotoxicity in rats. Basic \& clinical pharmacology \& toxicology, 100(3):145-150.

- AranyI,SafirsteinRL (2003). Cisplatin nephrotoxicity. In Seminars in nephrology,WB Saunders, 23( 5): 460-464.

- AyacheJ, BeaunierL, Boumendil J, Ehret G , LaubD(2010). Methodology: General Introduction. In Sample Preparation
SVU-IJMS, 5(1):331-342

Handbook for Transmission Electron Microscopy (2):1-2. Springer, New York, NY.

- BoonsanitD,KanchanapangkaS, Buranakarl C(2006). L-carnitine ameliorates doxorubicin-induced nephrotic syndrome in rats. Nephrology, 11(4): 313320.

- BurneM J, DanielsF, El Ghandour A, MauiyyediS, Colvin RB, O'Donnell M P, et al. (2001). Identification of the CD4+ $\mathrm{T}$ cell as a major pathogenic factor in ischemic acute renal failure. The Journal of clinical investigation, 108(9):1283-1290.

- CayirK, KaradenizA, YildirimA, Kalkan Y,KarakocA, Keles M, et al.(2009). Protective effect of L-carnitine against cisplatin-induced liver and kidney oxidant injury in rats. Central European Journal of Medicine, 4(2): 184-191.

- ChangB, Nishikawa M, SatoE, Utsumi K, InoueM(2002). L-Carnitine inhibits cisplatin-induced injury of the kidney and small intestine. Archives of Biochemistry and Biophysics, 405(1):55-64.

- FatenRA, IbrahimAE,Khaled AE(2013). Protective and modulatory effects of Curcumin and L-Carnitine against Methotrexate-induced Oxidative stress in albino rats. ResJ Pharm Biol. ChemSci (4): 744-754.

- Gamble M(2008). The hematoxylins and eosin. In:Bancroft JD, Gamble M, editors. Theory and practiceof histological techniques. 6th ed. London (UK):Churchill Livingston/Elsevier Inc. 121134.https://doi.org/10.1016/B978-0-44310279-0.50016-6.

- GuptaSC, PatchvaS, KohW,AggarwalBB (2012). Discovery of curcumin, a component of golden spice, and its miraculous biological activities. Clinical and experimental pharmacology and physiology, 39(3):283-299.

- Kumar P, Sulakhiya K, Barua CC,MundheN(2017). TNF- $\alpha$, IL-6 and IL10 expressions, responsible for disparity in 
action of curcumin against cisplatininduced nephrotoxicity in rats. Molecular and cellular biochemistry, 431(1):113-122.

- McSweeneyKR, GadanecLK, QaradakhiT, Ali BA, ZulliA,Apostolopoulos V (2021). Mechanisms of Cisplatin-Induced Acute Kidney Injury: Pathological Mechanisms, Pharmacological Interventions, and Genetic Mitigations. Cancers, 13(7):1572.

- Nameni F,Nuranipilehrud M (2019).Comparing the Effects of Curcumin Supplementation and that of L-Carnitine Supplementation on Superoxide Dismutase, Glutathione Peroxidase, Catalase and Glutathione Reductase after Acute Exercise. CMJA,8 (4):3517-3530.

- PablaN, Dong Z (2008).Cisplatin nephrotoxicity: mechanisms and renoprotective strategies. Kidney International, 73(9):994-1007.

- Prakobwong S, Gupta S C, Kim J H, SungB, PinlaorP, Hiraku Y, et al.(2011). Curcumin suppresses proliferation and induces apoptosis in human biliary cancer cells through modulation of multiple cell signaling

pathways. Carcinogenesis, 32(9):13721380.

- Ravindra P, Bhiwgade A, Kulkarni S, Rataboli P V,Dhume C Y (2010). Cisplatin induced histological changes in renal tissue of rat. Journal of Cell and Animal Biology, 4(7):108-111.

- Rizk A A, Shawky Y M,Motawie A G (2019). Can sildenafil citrate ameliorate cisplatin-induced nephrotoxicity? Crosstalk between the possible mechanisms. Eur janat, 23(2): 113-119.

- Sadeghi H, Mansourian M, PanahikokhdanE, SalehpourZ, Sadati I, Abbaszadeh-Goudarzi $\mathrm{K}$, et al. (2020). Antioxidant and protective effect of StachyspiliferaBenth against nephrotoxicity induced by cisplatin in rats. Journal of Food Biochemistry, 44(5): e13190.
- Sayed-Ahmed M M, Eissa M A, Kenawy S A, Mostafa N, Calvani M, OsmanAM $M$ (2004). Progression of cisplatin-induced nephrotoxicity in a carnitine-depleted rat model. Chemotherapy, 50(4): 162-170.

- Sohail N, Hira K, Kori J A, Farhat H, UroojF, Khan W, et al. (2021). Nephroprotective effect of ethanol extract and fractions of a sea lettuce, Ulvafasciata against cisplatin-induced kidney injury in rats. Environmental Science and Pollution Research, 28(8):9448-9461..

- Topcu-Tarladacalisir Y, Sapmaz-Metin M,Karaca T(2016).Curcumin counteracts cisplatin-induced nephrotoxicity by preventing renal tubular cell apoptosis. Renal Failure, 38(10):17411748.

- Trujillo J, Chirino YI, Molina-Jijón E, Andérica-Romero AC, Tapia E,Pedraza-Chaverrí J (2013).Renoprotective effect of the antioxidant curcumin: Recent findings. Redox biology, 1(1):448-456.

- Tufekci O, Gunes D, Ozoğul C, Kolatan E, Altun Z, Yilmaz O,et al. (2009). Evaluation of the effect of acetyl Lcarnitine on experimental cisplatin nephrotoxicity. Chemotherapy, 55(6): 451459. https://doi.org/10.1159/000240020

- UgurS, Ulu R, Dogukan A, Gurel A, Yigit IP,Gozel N, et al.(2015). The renoprotective effect of curcumin in cisplatin-induced nephrotoxicity. Renal Failure, 37(2):332-336.

- UzunogluS, Karagol H, Ozpuyan F, Cosar R, Cicin I, YurutcalogluV, et al.(2011). Protective effect of L-carnitine versus amifostine against cisplatin-induced nephrotoxicity in rats. Medical Oncology, 28(1): 690-696.

- Volarevic V, Djokovic B, Jankovic M G, Harrell C R, Fellabaum C, Djonov $\mathrm{V}$, et al. (2019). Molecular mechanisms of cisplatin-induced nephrotoxicity: a balance on the knife edge between renoprotection and tumor 
toxicity. Journal of Biomedical

Science, 26(1): 1-14.

- Waseem M, KaushikP,ParvezS(2013). Mitochondria-mediated mitigatory role of curcumin in cisplatin-induced nephrotoxicity. Cell Biochemistry and Function, 31(8):678-684.

- YaoX, Panichpisal K, KurtzmanN, NugentK (2007). Cisplatin nephrotoxicity: a review. The American Journal of the Medical Sciences, 334(2): 115-124.

- Yürekli Y, ̈̈nak P, Yenisey Ç, Ertay T, Müftüler F Z B,MedineE I (2011). Lcarnitine protection against cisplatin nephrotoxicity in rats: comparison with amifostin using quantitative renal Tc $99 \mathrm{~m}$ DMSA uptake. Molecular Imaging and Radionuclide Therapy, 20(1):1. 